\title{
A relação público-privada no SUS: um olhar sobre a assistência ambulatorial de média e alta complexidade na região de saúde da Baixada Cuiabana em Mato Grosso'
}

\author{
Cátia Maria Costa Romano \\ Escola de Enfermagem da Universidade Federal da Bahia \\ João Henrique Gurtler Scatena \\ Instituto de Saúde Coletiva da Universidade Federal de Mato Grosso
}

\begin{abstract}
O artigo objetiva discutir a relação público-privada na assistência ambulatorial de média e alta complexidade no SUS, na região da Baixada Cuiabana (MT). No Brasil, as imbricações entre os setores público e privado na saúde e suas consequências são complexas e ainda pouco estudadas. Este é um estudo quantitativo e descritivo, com base em dados secundários de Sistemas de Informação do SUS, referentes à estrutura, à produção e aos gastos desse tipo de assistência. Os resultados apontam que o número de estabelecimentos, a produção e o volume de recursos se concentram em Cuiabá, tendo o setor privado/filantrópico como principal provedor. Conclui-se que tal fato possa sugerir que esse setor detenha poder político e de pressão sobre o SUS, tanto na negociação de seus contratos quanto na prestação de serviços ambulatoriais mais bem remunerados.
\end{abstract}

Palavras-chave: Sistema Único de Saúde; serviços de saúde; assistência ambulatorial.

La relación público-privada en el SUS: una mirada a la atención ambulatoria de mediana y alta complejidad en la región sanitaria "Baixada Cuiabana" en Mato Grosso

El artículo aborda la colaboración público-privada en la atención ambulatoria secundaria y terciaria en el SUS, en la Baixada Cuiabana (MT). En Brasil, la superposición entre los sectores público y privado de la salud y sus consecuencias son complejas y aún poco estudiadas. Se trata de un estudio cuantitativo y descriptivo, con base en datos secundarios de los Sistemas de Información del SUS, en cuanto a la estructura, la producción y los costes de dicha asistencia. Los resultados muestran que el número de establecimientos, la producción y el volumen de los recursos están concentrados en Cuiabá, con el

http://dx.doi.org/10.1590/0034-76121618

Artigo recebido em 24 jun. 2013 e aceito em 10 jan. 2014.

1 Este trabalho faz parte da tese de doutorado do primeiro autor, intitulada: "A relação público-privada na atenção ambulatorial de média e alta complexidade do Sistema Único de Saúde na região da Baixada Cuiabana — MT", em desenvolvimento junto ao Programa de Pós-Graduação em Ciências da Saúde da Faculdade de Medicina da Universidade Federal de Mato Grosso (UFMT). Os autores agradecem ao CNPq (Proc. no 481893/2010-5) e à Capes (Procad-NF no 2275/2008) pelo apoio ao projeto em que este trabalho se insere. 
sector privado/filantrópico como principal proveedor. Este hecho podría sugerir que este sector tiene el poder político y la presión en el SUS, tanto en la negociación de sus contratos como en la prestación de servicios ambulatorios mejor pagados.

Palabras clave: Sistema Único de Salud; servicios de salud; atención ambulatoria.

The public-private relationship within SUS: a look upon ambulatory care of medium and high complexity in the health region called "Baixada Cuiabana", in the State of Mato Grosso

The article discusses the public-private relationship in ambulatory care of medium and high complexity within SUS, in the region of Baixada Cuiabana (MT). In Brazil, the overlapping between public and private sectors in the health system and its consequences are complex and still poorly studied. This is a quantitative and descriptive study, based on secondary data from the SUS Information Systems, concerning structure, production and costs of the aforementioned assistance. The results indicate that the number of establishments, production and volume of resources are concentrated in Cuiabá, and the private/philanthropic sector is the main provider. Such fact may suggest that this sector holds political power over SUS, both in contract negotiation and in the provision of better remunerated ambulatory services.

KEYWORDs: unified health system; health services; ambulatory care.

\section{Introdução}

A Constituição Brasileira de 1988 (Brasil, 1988), que instituiu o Sistema Único de Saúde (SUS), no âmbito da seguridade social, também assegurou em seu artigo 199 (§ 1ํ) a participação da iniciativa privada na provisão de serviços de saúde, desde que de forma complementar, sob as diretrizes e regras (contrato) deste sistema público. Tal fato possibilitou a manutenção de um espaço para o setor privado dentro do SUS gerando, muitas vezes, situações conflituosas na arena das negociações e pactuações com vistas à oferta de serviços de saúde, envolvendo gestores estaduais e municipais e também provedores privados de serviços específicos.

Corroborando essa afirmativa, Campos (2008) refere que o SUS assegurou a reprodução de grande parte do setor privado de prestação de serviços quando de sua criação, optando por não tornar estatais serviços privados, filantrópicos ou não governamentais que prestavam assistência ao antigo sistema previdenciário ou público. No sentido de integrá-los em rede única, delegou-se aos municípios, ou eventualmente aos estados, a função de realizar contratos e convênios com esses prestadores autônomos, acompanhando o seu desempenho.

Frequentemente, os gestores do SUS compram serviços de entidades privadas, o que reduz a capacidade desses gestores de intervir nas prioridades de atendimento e mesmo no fluxo de pessoas a serem atendidas. Além disso, o sistema de compras de serviços pode ter preços arbitrários, e também os prestadores privados tendem a selecionar casos e procedimentos rentáveis, recusando outros com menor lucratividade (Campos, 2008).

Faz-se necessária a contratualização dos serviços de saúde, objetivando melhor resposta às necessidades e expectativas de saúde da população, mediante prestação de serviços efetiva 
e de qualidade. Essa contratualização busca estabelecer uma relação clara e objetiva, definindo, a partir de um processo de negociação, metas e resultados a serem atingidos, sempre com foco nas necessidades de saúde da população. Dessa forma, o relacionamento de parceria entre as partes contratantes passa a ser mais importante do que o formalismo do contrato (Leite, Souza e Nascimento, 2010).

Solla (2006) refere que a grande maioria dos estados e municípios brasileiros contrata serviços privados sem cumprir a legislação vigente, não realizando, efetivamente, procedimentos licitatórios e não formalizando contratos com os prestadores. Tal fato dá a esse tipo de contratação uma aparência de "favor dos governantes" que, assim, poderiam receber retribuições dos contratados por esse "privilégio".

É quase universal a ausência de formalização da relação entre gestor público e unidades particulares de saúde (Matos e Pompeu, 2003). Segundo os autores, a percentagem desses contratos situa-se entre 10 e $20 \%$, dependendo do tipo de unidade. Um dos motivos para a baixa contratação dos estabelecimentos particulares de saúde são as indefinições presentes no processo de contratação. O setor público não desenvolveu a capacidade de formalização contratual de serviços privados de saúde, gerando uma situação em que se torna prisioneiro da oferta dos prestadores de serviços privados.

Diante dos inúmeros obstáculos enfrentados pela saúde pública brasileira, constata-se a dificuldade de criação e manutenção de um modelo de gestão pública no SUS que consiga executar serviços que atendam as necessidades da população de forma direta, sem a complementação do setor privado.

No contexto atual do SUS, se a atividade privada deve conviver com a pública no mesmo espaço social de garantias de direitos, a privada complementando a pública, mediante ajuste de colaboração e contratos de prestação de serviços, é necessário encarar essa realidade e regulá-la em prol do interesse público. Eficiência e bom desempenho não são particularidades exclusivas do setor público e tampouco do setor privado. Ambos são importantes para o SUS e a polarização da discussão público versus privado não contribui para a melhoria da gestão pública da saúde (Santos, 2010).

Não é comum que as imbricações entre os dois sistemas (público/privado) sejam consideradas, e muito menos as consequências da existência de um sistema dual para o financiamento, a eficácia da assistência e a sustentabilidade política do sistema público, o que pode significar que não estão sendo formatadas propostas de políticas públicas alternativas para o setor (Menicucci, 2010).

Torna-se ainda mais grave essa situação quando se constata a escassez de estudos sobre a privatização da rede assistencial do SUS e as relações entre o público e o privado no Brasil (Bahia, 2008). Tal fato também ocorre no estado de Mato Grosso, o que salienta a importância desta investigação.

Pretende-se, neste artigo, discutir a relação público-privada no SUS, numa região de saúde específica deste estado, a partir da caracterização da estrutura da rede de assistência ambulatorial de média e alta complexidade, bem como da produção dessa assistência e dos valores aprovados. 


\section{Método}

Este é um estudo de natureza quantitativa, exploratório e descritivo. Para apreensão de informações referentes à assistência ambulatorial de média e alta complexidade, foram utilizados dados secundários provenientes do Cadastro Nacional de Estabelecimentos de Saúde (Cnes) e do Sistema de Informações Ambulatoriais (SIA-SUS), tendo como recorte temporal o ano de 2011. Esses dados foram apreendidos on-line do sítio do Datasus/MS.

$\mathrm{Na}$ base de dados do CNES, para o levantamento dos estabelecimentos prestadores de assistência ambulatorial e de Serviço de Apoio Diagnóstico e Terapêutico (Sadt) de média e alta complexidade do SUS foram utilizadas duas variáveis: Tipo de estabelecimento e Tipo de prestador. Quanto ao tipo de estabelecimento foram selecionados: Centro de Atenção Hemoterápica; Clínica/Ambulatório Especializado; Hospital Especializado; Hospital Geral; Laboratório Central (Lacen); Policlínica; e Unidade de Serviço de Apoio Diagnóstico e Terapêutico (Sadt). Os serviços hospitalares foram selecionados porque neles também há unidades ambulatoriais. Cabe salientar que alguns Sadt, da mesma forma, realizam atendimento ambulatorial, além dos serviços de diagnóstico e terapia. Da variável tipo de prestador foram selecionados prestadores: públicos, agregando instituições federais, estaduais e municipais; privados, agregando instituições privadas com fins lucrativos e sem fins lucrativos; e filantrópicos.

Objetivando caracterizar a produção e o valor aprovados dos serviços ambulatoriais de média e alta complexidade, utilizou-se a base de dados do SIA/SUS selecionando as variáveis: quantidade aprovada e valor aprovado. Ambas as variáveis foram analisadas por municípios da região da Baixada Cuiabana e por tipo de prestador.

Foram considerados procedimentos de média e alta complexidade todos aqueles que assim são classificados pelo SIA-SUS na seleção "Complexidade", com uma ressalva: nos procedimentos de alta complexidade, não foi incluído o grupo "medicamentos" (grupo de procedimentos 6), tendo em vista que eles somente são disponibilizados na capital, sob responsabilidade única do setor público.

O estado de Mato Grosso (MT) possui 141 municípios e encontra-se dividido em 16 regiões de saúde. O lócus desta pesquisa é a região da Baixada Cuiabana, com população de 911.482 habitantes. Cuiabá, capital de MT e município sede dessa região, é o mais populoso do estado (551.098 hab.). Juntamente com Várzea Grande (252.596 hab.), os dois municípios concentram 88\% da população dessa região (IBGE, 2010).

A Baixada Cuiabana é uma região que desde o final dos anos 1980 manteve-se quase inalterada, em termos espaciais. Sua conformação obedeceu a critérios que Viana e colaboradores (2010) salientam como importantes na constituição de uma região: contiguidade, facilidade de acesso, capacidade instalada, capacidade de gestão e capacidade técnico-política de seus municípios. Atualmente, a região engloba 11 municípios: Acorizal; Barão de Melgaço; Chapada dos Guimarães; Cuiabá; Jangada; Nossa Sra. Livramento; Nova Brasilândia; Planalto da Serra; Poconé; S. Antônio do Leverger e Várzea Grande. 
Os dados aqui analisados foram extraídos de sistemas de informações públicos e de acesso universal. No entanto, eles são parte de um projeto maior, aprovado pelo Comitê de Ética em Pesquisa do Hospital Universitário Júlio Müller (Protocolo no 681/CEP-HUJM/09 e Of. 079/CEP-HUJM/2011) e que cumpriu todas as normas éticas estabelecidas.

\section{Resultados e discussão}

Quando se analisam os estabelecimentos prestadores de Assistência Ambulatorial na Baixada Cuiabana (tabela 1), observa-se que a maioria deles está vinculada ao setor público $(61,3 \%)$, enquanto os prestadores privados/filantrópicos são responsáveis por 38,7\% dessa provisão.

A situação praticamente se inverte quando são investigados os estabelecimentos que prestam Serviços de Apoio Diagnóstico e Terapia (Sadt). Os prestadores privados/filantrópicos do SUS detêm 65,5\% desses estabelecimentos, enquanto os prestadores públicos são responsáveis por $34,5 \%$ deles.

\section{Tabela 1}

Distribuição de estabelecimentos prestadores de assistência ambulatorial e de Sadt de média e alta complexidade no SUS, segundo tipo de prestador, Região de Saúde da Baixada Cuiabana-MT, 2011

\begin{tabular}{|c|c|c|c|c|c|c|c|c|}
\hline \multirow{3}{*}{ Tipo de Estabelecimento } & \multicolumn{6}{|c|}{ Tipo de prestador } & \multirow{2}{*}{\multicolumn{2}{|c|}{ Total }} \\
\hline & \multicolumn{2}{|c|}{ Público } & \multicolumn{2}{|c|}{ Privado } & \multicolumn{2}{|c|}{ Filantrópico } & & \\
\hline & № & $\%$ & № & $\%$ & № & $\%$ & № & $\%$ \\
\hline Ass. Ambulatorial & 57 & 61,3 & 29 & 31,2 & 7 & 7,5 & 93 & 100 \\
\hline Sadt & 29 & 34,5 & 50 & 59,5 & 5 & 6,0 & 84 & 100 \\
\hline
\end{tabular}

Fonte: Elaboração própria com base em dados do Cadastro Nacional de Estabelecimentos de Saúde (Cnes/SUS).

Considerando apenas o número de estabelecimentos e não o que eles produzem, pode-se dizer, pelo pequeno percentual que eles representam - 7,5\% na Assistência Ambulatorial e $6 \%$ dos Sadt —, que os prestadores filantrópicos têm um papel complementar, respeitando o que preceitua o art. 199 (§ 1ํ) da Constituição de 1988.

$\S 1^{\circ}$ As instituições privadas poderão participar de forma complementar do sistema único de saúde, segundo diretrizes deste, mediante contrato de direito público ou convênio, tendo preferência as entidades filantrópicas e as sem fins lucrativos. 
É importante salientar que é grande o número de estabelecimentos privados e filantrópicos existentes nesta região de saúde e apenas uma pequena parcela deles presta serviços ao SUS. Não estão vinculados ao SUS $88,3 \%$ dos estabelecimentos prestadores de assistência ambulatorial e 73,8\% dos prestadores de Sadt (tabela 2).

O setor privado/filantrópico que está desvinculado do SUS não é objeto de investigação deste trabalho, ao menos não diretamente. No entanto, considera-se importante conhecer a dimensão desse segmento, pois ela mostra a "reserva de mercado" que esse setor representa nesse tipo de assistência. Não se pode descartar a possibilidade da força do setor privado/filantrópico ser um dos elementos que têm peso no processo de negociação entre o SUS e seus prestadores, no que tange a credenciamentos, contratualizações, pagamentos diferenciados da tabela SUS por serviço e mesmo ao atendimento de demandas autorizadas por liminares judiciais.

Tabela 2

Distribuição de estabelecimentos privados e filantrópicos prestadores de assistência ambulatorial e de Sadt de média e alta complexidade no SUS, segundo vínculo com o SUS, Região de Saúde da Baixada Cuiabana (MT), 2011

\begin{tabular}{|c|c|c|c|c|c|c|}
\hline \multirow{2}{*}{ Tipo de Estabelecimento } & \multicolumn{2}{|c|}{ Não vinculados ao SUS } & \multicolumn{2}{|c|}{ Vinculados ao SUS } & \multicolumn{2}{|c|}{ Total } \\
\hline & № & $\%$ & № & $\%$ & № & $\%$ \\
\hline Ass. Ambulatorial & 265 & 88,3 & 35 & 11,7 & 300 & 100 \\
\hline Sadt & 149 & 73,8 & 53 & 26,2 & 202 & 100 \\
\hline
\end{tabular}

Fonte: Elaboração própria com base em dados do Cadastro Nacional de Estabelecimentos de Saúde (CNES).

Quando é investigada, entre os municípios da região, a distribuição dos estabelecimentos que prestam atendimento ambulatorial de média e alta complexidade no SUS, observa-se que somente em Cuiabá a participação do setor privado/filantrópico é expressiva (51,6\%), enquanto em Várzea Grande tal percentual é de $26,7 \%$ (tabela 3). Já entre os demais municípios da região, a totalidade de estabelecimentos prestadores desse tipo de assistência pertence ao setor público. Observa-se que é grande a concentração desses estabelecimentos em Cuiabá $(62 / 93=66,7 \%)$, mas ela é bem maior quando só se consideram os estabelecimentos privados/filantrópicos, correspondendo a 88,8\% (32/36).

Em relação aos estabelecimentos que prestam Sadt, dos 11 municípios que constituem a Baixada Cuiabana, quatro deles não os possuem: Acorizal, Barão de Melgaço, Nova Brasilândia e Planalto da Serra. Cuiabá concentra 70,2\% (59/84) dos estabelecimentos dessa região, e sua maioria $(74,6 \%)$ pertence ao setor privado/filantrópico. Várzea Grande concentra $23,8 \%$ (20/84) desses serviços, que estão distribuídos equitativamente entre ambos os setores, público e privado/filantrópico (tabela 4). 
Tabela 3

Distribuição de estabelecimentos prestadores de assistência ambulatorial de média e alta complexidade no SUS, segundo município e tipo de prestador, Região de Saúde da Baixada Cuiabana (MT), 2011

\begin{tabular}{|c|c|c|c|c|c|c|c|c|}
\hline \multirow{3}{*}{ Município } & \multicolumn{6}{|c|}{ Tipo de Prestador } & \multirow{2}{*}{\multicolumn{2}{|c|}{ Total }} \\
\hline & \multicolumn{2}{|c|}{ Público } & \multicolumn{2}{|c|}{ Privado } & \multicolumn{2}{|c|}{ Filantrópico } & & \\
\hline & № & $\%$ & $\stackrel{\circ}{0}$ & $\%$ & № & $\%$ & № & $\%$ \\
\hline Acorizal & 1 & 100 & - & - & - & - & 1 & 100 \\
\hline Barão de Melgaço & 2 & 100 & - & - & - & - & 2 & 100 \\
\hline Chapada dos Guimarães & 2 & 100 & - & - & - & - & 2 & 100 \\
\hline Cuiabá & 30 & 48,4 & 25 & 40,3 & 7 & 11,3 & 62 & 100 \\
\hline Jangada & 1 & 100 & - & - & - & - & 1 & 100 \\
\hline Nossa Sra. Livramento & 2 & 100 & - & - & - & - & 2 & 100 \\
\hline Nova Brasilândia & 1 & 100 & - & - & - & - & 1 & 100 \\
\hline Planalto da Serra & 2 & 100 & - & - & - & - & 2 & 100 \\
\hline Poconé & 3 & 100 & - & - & - & - & 3 & 100 \\
\hline S. Antônio do Leverger & 2 & 100 & - & - & - & - & 2 & 100 \\
\hline Várzea Grande & 11 & 73,3 & 4 & 26,7 & - & - & 15 & 100 \\
\hline Total & 57 & 61,3 & 29 & 31,2 & 7 & 7,5 & 93 & 100 \\
\hline
\end{tabular}

Fonte: Elaboração própria com base em dados do Cadastro Nacional de Estabelecimentos de Saúde (Cnes).

Em determinados municípios e regiões a oferta ambulatorial especializada ainda se encontra fortemente vinculada aos serviços privados (filantrópicos ou lucrativos). A contratação desses serviços pelo SUS tende a oferecer certa agilidade e ampliar a resolutividade para os usuários que conseguem ter acesso. Porém, têm-se observado algumas distorções importantes em serviços ambulatoriais oferecidos pelos estabelecimentos privados, em particular quando não estão sujeitos aos mecanismos de regulação e controle. Como exemplo disso tem-se: a seleção de demanda por enfermidades que resultam em procedimentos mais bem remunerados segundo a tabela SUS, o excesso de pedidos de consultas com outros especialistas e de exames complementares, muitas vezes desnecessários, ou ainda a utilização desses serviços para a realização de procedimentos não cobertos pelos planos de saúde ou não autorizados pelas operadoras (Solla e Chioro, 2008, 2012). 
Tabela 4

Distribuição de estabelecimentos prestadores de Sadt de média e alta complexidade no SUS, segundo município e tipo de prestador, Região de Saúde da Baixada Cuiabana (MT), 2011

\begin{tabular}{|c|c|c|c|c|c|c|c|c|}
\hline \multirow{3}{*}{ Município } & \multicolumn{6}{|c|}{ Tipo de Prestador } & \multirow{2}{*}{\multicolumn{2}{|c|}{ Total }} \\
\hline & \multicolumn{2}{|c|}{ Público } & \multicolumn{2}{|c|}{ Privado } & \multicolumn{2}{|c|}{ Filantrópico } & & \\
\hline & № & $\%$ & № & $\%$ & № & $\%$ & № & $\%$ \\
\hline Chapada dos Guimarães & 1 & 100 & - & - & - & - & 1 & 100 \\
\hline Cuiabá & 15 & 25,4 & 40 & 67,8 & 4 & 6,8 & 59 & 100 \\
\hline Jangada & 1 & 100 & - & - & - & - & 1 & 100 \\
\hline Nossa Sra. Livramento & 1 & 100 & - & - & - & - & 1 & 100 \\
\hline Poconé & - & - & - & - & 1 & 100 & 1 & 100 \\
\hline S. Antônio do Leverger & 1 & 100 & - & - & - & - & 1 & 100 \\
\hline Várzea Grande & 10 & 50,0 & 10 & 50,0 & - & - & 20 & 100 \\
\hline Total & 29 & 34,5 & 50 & 59,5 & 5 & 6,0 & 84 & 100 \\
\hline
\end{tabular}

Fonte: Elaboração própria com base em dados do Cadastro Nacional de Estabelecimentos de Saúde (Cnes).

Em determinados municípios e regiões a oferta ambulatorial especializada ainda se encontra fortemente vinculada aos serviços privados (filantrópicos ou lucrativos). A contratação desses serviços pelo SUS tende a oferecer certa agilidade e ampliar a resolutividade para os usuários que conseguem ter acesso. Porém, têm-se observado algumas distorções importantes em serviços ambulatoriais oferecidos pelos estabelecimentos privados, em particular quando não estão sujeitos aos mecanismos de regulação e controle. Como exemplo disso tem-se: a seleção de demanda por enfermidades que resultam em procedimentos mais bem remunerados segundo a tabela SUS, o excesso de pedidos de consultas com outros especialistas e de exames complementares, muitas vezes desnecessários, ou ainda a utilização desses serviços para a realização de procedimentos não cobertos pelos planos de saúde ou não autorizados pelas operadoras (Solla e Chioro, 2008, 2012).

Globekner (2011), reforçando o exposto pelos autores acima, afirma a existência da seleção entre serviços que são demandados para o setor público, na maioria das vezes, os menos onerosos.

A situação apresentada na região da Baixada Cuiabana é semelhante ao que é observado em todo o país. Segundo Noronha, Santos e Pereira (2010), as diversas modalidades de serviços que compõem o sistema de saúde não se distribuem uniformemente no Brasil. Grande parte dos municípios é muito pequena e não possui demanda suficiente para abrigar todos os níveis de atenção à saúde em seu território. A maioria deles conta apenas com serviços de atenção básica, necessitando de serviços de outros municípios, para atender níveis complementares dessa atenção. Nesse cenário, a disponibilidade de serviços especializados e de alta complexidade no SUS está bastante concentrada em apenas alguns municípios. 
Na Baixada Cuiabana, os estabelecimentos prestadores de assistência ambulatorial de média e alta complexidade concentram-se em Cuiabá, município também considerado referência para tal tipo de assistência no estado de Mato Grosso. Salienta-se que a maioria dos municípios que compõem esta região tem população inferior a 20 mil habitantes, assim como a maior parte dos municípios mato-grossenses. Em Cuiabá a participação do setor privado/filantrópico do SUS é pouco maior do que a de prestadores públicos, na provisão da assistência ambulatorial (51,6\%), mas é majoritária na provisão dos Sadt (74,6\%), conforme evidenciado na tabela 3. No município de Cuiabá, a participação dos setores privado e filantrópico no SUS, em número de estabelecimentos, é maior do que a do setor público, colocando em xeque a questão da complementaridade da iniciativa privada no SUS.

A Constituição Brasileira de 1988 tratou desse tema ao permitir ao poder público recorrer aos serviços privados de saúde quando os seus próprios fossem insuficientes (Santos, 2010).

Cabe salientar que, no ano de 2011, 40 municípios do estado não tinham estabelecimentos com atendimentos de Sadt. Dos demais 101 municípios, Cuiabá concentrou quase 20\% desses estabelecimentos, enquanto 51 municípios concentraram 0,3\% cada (Cnes, 2012).

A existência ou não de estabelecimentos provedores de serviços especializados em determinado município não necessariamente significa acesso a essa assistência, pelo conjunto da população. É, no entanto, um indício, assim como sua distribuição, de problemas de acesso. Neste sentido, reitera-se a necessidade de melhorar a regulação, organizando fluxos adequados a todos os níveis de complexidade dos serviços que compõem o sistema de saúde (Pustai, 2004).

Para Santos e Merhy (2006), a regulação pode ser entendida como a capacidade de intervir nos processos de prestação de serviços, alterando ou orientando sua execução. Essa intervenção pode ser feita por intermédio de mecanismos indutores, normalizadores, regulamentadores ou restritores. A intervenção entre a demanda e a prestação direta dos serviços, em seus diversos aspectos, pode ser caracterizada como mecanismo de regulação.

Padrões adequados de acesso, resolutividade e excelência na qualidade da prestação do serviço e acolhimento são um desafio para os sistemas nacionais de saúde, principalmente aqueles que têm forte presença estatal no provimento da assistência, como é o caso do SUS. Sob o prisma da equidade na assistência à saúde, pode-se inferir que a regulação é imprescindível e sua finalidade é assegurar que se atinjam os grandes objetivos sociais do sistema de saúde (Farias et al., 2011).

A necessidade de regulação é ainda mais evidente nos municípios-polo, uma vez que nesses locais a demanda para os serviços especializados pode ser maior do que a da atenção básica, pois além dos habitantes do município, outros, referenciados da área da abrangência regional, somam-se aos usuários locais. Afora isso, frequentemente, esses serviços recebem usuários do sistema de saúde suplementar, principalmente daquelas operadoras que disponibilizam coberturas parciais para determinados procedimentos, normalmente os mais caros, pressionando esses usuários a procurarem os serviços do SUS, o que amplia as distorções na utilização dos mesmos (Silva, 1996). 
A precária integração entre os serviços de saúde aparece como uma característica presente na maioria dos sistemas de saúde das Américas por várias décadas, gerando a fragmentação e duplicação de serviços. Estudos da Organização Pan-Americana de Saúde e da Organização Mundial de Saúde evidenciam que tal insuficiência vem ocasionando redução na qualidade e efetividade da rede assistencial, aumentando desnecessariamente os custos da atenção à saúde, ampliando as desigualdades no acesso e gerando grande ineficiência do sistema como um todo (Solla e Chioro, 2012).

Os mesmos autores referem que, em função destes aspectos, as reformas do setor de saúde realizadas em muitos países nesta região têm colocado a necessidade de efetivar a integração entre os diferentes níveis de atenção; contudo, poucos têm conseguido sucesso.

A inviabilidade, também em termos de economia de escala, de se manter serviços especializados em municípios pequenos com baixa demanda para os mesmos é um dos fatores que remetem à necessidade da organização do sistema em redes de atenção e de um sistema de regulação bem definido quanto às suas normas operacionais entre o estado e os municípios das regiões de saúde (Mendes, 2011).

O conjunto de redes de atenção, bem articulado, pressupõe uma adequada integração, colocando sob mesma gestão diferentes pontos de atenção em um mesmo sistema de saúde, viabilizando a continuidade da atenção aos usuários do sistema e a integralidade do cuidado a ser prestado. Necessário se faz apontar que essa integração deve ocorrer a partir da atenção básica, principal porta de entrada do sistema (a outra correspondendo aos serviços de urgência e emergência), ordenando a necessidade de definição, pelo sistema, de fluxos e contrafluxos, referências e contrarreferências. Entre as principais vantagens consentidas por esta integração, incluem-se a melhoria da qualidade da atenção, a redução de custos com economia de escala e o aumento da eficiência do sistema (Mendes, 2001, 2011; Santos, 2013).

Fleury (2002) também compreende a necessidade da organização em redes dos serviços sociais caracterizando-se em ações e serviços coordenados, espalhados por diversos entes, conseguindo manter a unidade em diferentes formas de estruturas político-jurídicas, administrativas, técnicas e territoriais.

Nessa linha, Santos (2013) adverte que rede não é o simples agrupamento de serviços ou organizações, mas pressupõe a adoção de aspectos que deem sentido a esse entrelaçamento de ações e processos, garantindo uma institucionalidade, uma organicidade à rede, objetivando a otimização de seus recursos e meios.

Para Davanzo e Fonseca (2002), a organização de uma rede regionalizada e hierarquizada é imprescindível para garantir a atenção especializada, tornando-se necessário o reconhecimento de recortes espaciais demarcando um conjunto de municípios, com hierarquia estabelecida de acordo com a capacidade de ofertar determinadas ações e serviços especializados, racionalizando, deste modo, a atenção especializada, o que possibilita assegurar que todos os usuários tenham acesso aos procedimentos de saúde necessários.

Cavalcanti (2011) reforça o exposto quando explica que a fragilidade da capacidade de gerar assistência integral por parte dos municípios, quando isolados, fortaleceu a concepção de regiões de saúde como estratégia de atenção no SUS. 
No que tange à produção ambulatorial, foi possível diferenciá-la por nível de complexidade (média e alta). Em relação à produção ambulatorial de média complexidade, observa-se uma concentração $(73,2 \%)$ no setor público (tabela 5). Cabe salientar que, dos municípios dessa região, apenas Cuiabá e Várzea Grande (conurbação também conhecida como Grande Cuiabá) apresentam produção ambulatorial de média complexidade no setor privado do SUS, com $32,1 \%$ e $2 \%$, respectivamente.

\section{Tabela 5}

Distribuição da produção ambulatorial de média complexidade (quantidade aprovada), segundo município e tipo de prestador, Região de Saúde da Baixada Cuiabana (MT), 2011

\begin{tabular}{|c|c|c|c|c|c|c|c|c|}
\hline \multirow{3}{*}{ Município } & \multicolumn{6}{|c|}{ Tipo de Prestador } & \multirow{2}{*}{\multicolumn{2}{|c|}{ Total }} \\
\hline & \multicolumn{2}{|c|}{ Público } & \multicolumn{2}{|c|}{ Privado } & \multicolumn{2}{|c|}{ Filantrópico } & & \\
\hline & № & $\%$ & № & $\%$ & № & $\%$ & № & $\%$ \\
\hline Acorizal & 28.564 & 100 & - & - & - & - & 28.564 & 100 \\
\hline Barão de Melgaço & 10.688 & 100 & - & - & - & - & 10.688 & 100 \\
\hline Chapada Guimarães & 54.087 & 100 & - & - & - & - & 54.087 & 100 \\
\hline Cuiabá & 371.454 & 67,9 & 295.444 & 5,4 & 146.0651 & 26,7 & 5.470 .637 & 100 \\
\hline Jangada & 12.950 & 100 & - & - & - & - & 12.950 & 100 \\
\hline Nossa Sra. Livramento & 22.117 & 100 & - & - & - & - & 22.117 & 100 \\
\hline Nova Brasilândia & 13.599 & 100 & - & - & - & - & 13.599 & 100 \\
\hline Planalto da Serra & 4.675 & 100 & - & - & - & - & 4.675 & 100 \\
\hline Poconé & 108.086 & 100 & - & - & - & - & 108.086 & 100 \\
\hline S. Antônio Leverger & 9.301 & 100 & - & - & - & - & 9301 & 100 \\
\hline Várzea Grande & 850.513 & 98,0 & 17.707 & 2,0 & - & - & 868.220 & 100 \\
\hline Total da Produção & 4.829 .122 & 73,2 & 313.151 & 4,7 & 146.0651 & 22,1 & 6.602 .924 & 100 \\
\hline
\end{tabular}

Fonte: Elaboração própria com base em dados do Sistema de Informações Ambulatoriais do SUS (SIA/SUS).

A maior parcela dos valores aprovados para produção ambulatorial de média complexidade do SUS concentra-se no setor público $(69,9 \%)$, percentual discretamente inferior àquele relativo à produção (tabela 6). O setor privado do SUS foi responsável por 30,1\% das despesas com esse tipo de assistência, percentual discretamente superior àquele relativo à produção. Tal disparidade, ainda que pequena, indica que os procedimentos realizados pelo setor privado são um pouco mais dispendiosos para o SUS. Essa disparidade é mais evidente em Várzea Grande, onde, embora o setor privado concentre $2 \%$ da produção ambulatorial de média complexidade, ele consome 10,7\% dos valores pagos por essa assistência, ou seja, neste município esse setor realiza procedimentos mais caros do que aqueles feitos pelo setor público.

Geralmente, o campo da média complexidade vem sendo compreendido, no âmbito do SUS, apenas como lócus de produção dos serviços de apoio diagnóstico e terapêutico de atenção especializada de menor complexidade tecnológica ou, mais restritivamente, pelos 
procedimentos especializados de menor valor financeiro incluídos nas tabelas do SUS (Solla e Chioro, 2008, 2012).

A reflexão destes autores poderia explicar a participação maior do setor público na produção de média complexidade na Grande Cuiabá, uma vez que os valores pagos pela tabela do SUS, para procedimentos dessa natureza, provavelmente não sejam atrativos para o setor privado.

Tabela 6

Distribuição dos valores aprovados (em milhares de reais), referentes à assistência ambulatorial de média complexidade, segundo município e tipo de prestador, Região de Saúde da Baixada Cuiabana (MT), 2011

\begin{tabular}{|c|c|c|c|c|c|c|c|c|}
\hline \multirow{3}{*}{ Município } & \multicolumn{6}{|c|}{ Tipo de Prestador } & \multirow{2}{*}{\multicolumn{2}{|c|}{ Total }} \\
\hline & \multicolumn{2}{|c|}{ Público } & \multicolumn{2}{|c|}{ Privado } & \multicolumn{2}{|c|}{ Filantrópico } & & \\
\hline & № & $\%$ & № & $\%$ & № & $\%$ & № & $\%$ \\
\hline Acorizal & 87,903 & 100 & - & - & - & - & 87,903 & 100 \\
\hline Barão Melgaço & 34,135 & 100 & - & - & - & - & 34,135 & 100 \\
\hline Chapada Guimarães & 300,578 & 100 & - & - & - & -9 & 300,578 & 100 \\
\hline Cuiabá & $30.555,081$ & 66,3 & $5.271,409$ & 11,4 & $10.243,049$ & 22,3 & $46.069,541$ & 100 \\
\hline Jangada & 45,686 & 100 & - & - & - & - & 45,686 & 100 \\
\hline Nossa Sra. Livramento & 103,208 & 100 & - & - & - & - & 103,208 & 100 \\
\hline Nova Brasilândia & 65,143 & 100 & - & - & - & - & 65,143 & 100 \\
\hline Planalto da Serra & 20,655 & 100 & - & - & - & - & 20,655 & 100 \\
\hline Poconé & 679,729 & 100 & - & - & - & - & 679,729 & 100 \\
\hline S. Antônio Leverger & 64,826 & 100 & - & - & - & - & 64,826 & 100 \\
\hline Várzea Grande & $5.619,774$ & 89,3 & 673,453 & 10,7 & - & - & $6.293,228$ & 100 \\
\hline Total de despesas & $37.576,724$ & 69,9 & $5.944,862$ & 11,1 & $10.243,049$ & 19,0 & $53.764,637$ & 100 \\
\hline
\end{tabular}

Fonte: Elaboração própria com base em dados do Sistema de Informações Ambulatoriais do SUS (SIA/SUS).

No Brasil, a oferta de serviços de média complexidade é um dos pontos de maior estrangulamento do sistema de saúde e um dos principais entraves para garantir a integralidade da atenção (Solla e Chioro, 2012; Spedo, 2009). Historicamente, esses serviços têm sido organizados e financiados com base na lógica de oferta de procedimentos, desconsiderando as necessidades e o perfil epidemiológico da população (Solla e Chioro, 2012).

Tal lógica deve-se à imprecisão conceitual, quando, frequentemente, as ações de média complexidade são definidas por exclusão, ou seja, elas não cabem à Atenção Básica e tampouco estão classificadas na Alta Complexidade. Tais conceitos indicam uma visão fragmentária de sistema e ainda centrada na produção de procedimentos (Conass, 2007; Gottems et al., 2008).

Desse modo, a demanda acaba sendo condicionada pela oferta dos serviços existentes. Além disso, existe uma baixa capacidade de regulação sobre essa oferta e grande dificuldade 
na aquisição de serviços em função da defasagem dos valores pagos pelo SUS (Solla e Chioro, 2008, 2012).

Corroborando o explicitado acima, Mendes (2004) salienta que o SUS vem sendo constituído por meio de um enfoque na gestão da oferta dos serviços de saúde. Para o autor, a experiência internacional de reformas sanitárias evidencia a inferioridade dos sistemas planejados com base na oferta de serviços, em relação àqueles orientados pela demanda.

No Brasil, todas as normas operacionais do SUS acolheram e consolidaram o foco na oferta quando vincularam a habilitação dos municípios à existência física dos equipamentos de saúde em seu território (Mendes, 2004).

De outra forma, o foco na oferta está determinado pela sistemática de financiamento que se materializa na estratégia de o "dinheiro seguir o gestor". Isso significa que os recursos para a atenção à saúde dos cidadãos dos municípios satélites são transferidos para o gestor do município onde os serviços estão localizados. É esse foco na oferta dos serviços de saúde que gera uma normalização complexa, estabelece fluxos complicados das pessoas e dos recursos financeiros e dificulta o pronto acesso (Mendes, 2004).

Desse modo, os serviços de média complexidade caracterizam-se, normalmente, pela dificuldade de acesso e baixa resolubilidade, superposição de ofertas de serviços nas redes ambulatorial e hospitalar, concentração em locais de alta densidade populacional e baixo grau de integração entre as ações de diferentes níveis e graus de complexidade da assistência (Solla e Chioro, 2008).

Quanto à produção ambulatorial de alta complexidade na Baixada Cuiabana, ela se dá apenas na Grande Cuiabá, com 88,1\% dessa produção se dando no setor privado (tabela 7). Cuiabá concentra 94,3\% (277.786/294.695) da produção regional, com 87,5\% da mesma feitos pelo setor privado/filantrópico do SUS. Embora Várzea Grande responda por 5,7\% (16.909/294.695) desses procedimentos, praticamente todos eles (99\%) são providos pelo setor privado.

No que se refere aos valores aprovados com os procedimentos de alta complexidade, 97\% são relativos ao setor privado e apenas 3\% ao setor público (tabela 7). Tanto em Cuiabá como em Várzea Grande é elevada (acima de 96\%) a concentração desse valor no setor privado.

Além de deter 70\% dos estabelecimentos prestadores de Sadt da região (tabela 3), o município de Cuiabá concentra 94\% dos procedimentos aprovados de alta complexidade e 93\% de todo o valor aprovado com tais procedimentos, sendo 97\% desse valor destinados ao setor privado/filantrópico do SUS (tabela 7). Pelo menos no que tange aos valores aprovados, pode-se afirmar que a quase totalidade dos dispêndios do SUS com a alta complexidade, na Baixada Cuiabana, é canalizada para os provedores privados/filantrópicos de Cuiabá.

As evidências apresentadas podem sinalizar a existência de uma política de fortalecimento do setor privado no âmbito do SUS, em Mato Grosso. Fato esse fortemente caracterizado pela trajetória das políticas públicas de saúde no Brasil.

Historicamente, privilegiou-se no Brasil o setor privado, tornando-se clara essa opção com a criação do Instituto Nacional de Assistência Médica da Previdência Social, na metade do século passado, e a consequente expansão de rede privada (Bahia,1999; Faveret Filho e Oliveira, 1990) 
e dos serviços de atenção especializada, em particular, os de alta complexidade. Os interesses privados acabam determinando o padrão de oferta para o sistema, pois, de uma forma geral, os serviços públicos não possuem capacidade física instalada suficiente para suprir as necessidades de atenção especializada dos usuários do SUS (Solla e Chioro, 2008, 2012).

Tabela 7

Distribuição da produção ambulatorial de alta complexidade (quantidade aprovada) e dos valores aprovados (em milhares de reais), segundo município e tipo de prestador, Região de Saúde da Baixada Cuiabana (MT), 2011

\begin{tabular}{|c|c|c|c|c|c|c|c|c|}
\hline \multirow{3}{*}{ Produção por município } & \multicolumn{6}{|c|}{ Tipo de Prestador } & \multirow{2}{*}{\multicolumn{2}{|c|}{ Total }} \\
\hline & \multicolumn{2}{|c|}{ Público } & \multicolumn{2}{|c|}{ Privado } & \multicolumn{2}{|c|}{ Filantrópico } & & \\
\hline & № & $\%$ & № & $\%$ & № & $\%$ & № & $\%$ \\
\hline Cuiabá & 34.811 & 12,5 & 86.116 & 31,0 & 156.859 & 56,5 & 277.786 & 100 \\
\hline Várzea Grande & 173 & 1,0 & 16.736 & 99,0 & - & - & 16.909 & 100 \\
\hline Total da produção & 34.984 & 11,9 & 102.852 & 34,9 & 156.859 & 53,2 & 294.695 & 100 \\
\hline \multirow{2}{*}{$\begin{array}{l}\text { Valor aprovado por } \\
\text { município }\end{array}$} & \multicolumn{2}{|c|}{ Público } & \multicolumn{2}{|c|}{ Privado } & \multicolumn{2}{|c|}{ Filantrópico } & \multicolumn{2}{|l|}{ Total } \\
\hline & $\mathrm{N}^{\circ}$ & $\%$ & $\mathrm{~N}^{\circ}$ & $\%$ & $\mathrm{~N}^{\circ}$ & $\%$ & $\mathrm{~N}^{\circ}$ & $\%$ \\
\hline Cuiabá & $1.180,203$ & 3,1 & $15.258,676$ & 40,0 & $21.754,992$ & 56,9 & $38.193,871$ & 100 \\
\hline Várzea Grande & 17,097 & 0,6 & $2.848,454$ & 99,4 & - & - & $2.865,551$ & 100 \\
\hline Total das despesas & $1.197,301$ & 3,0 & $18.107,130$ & 44,1 & $21.754,992$ & 52,9 & $41.059,424$ & 100 \\
\hline
\end{tabular}

Fonte: Elaboração própria com base em dados do Sistema de Informações Ambulatoriais do SUS (SIA/SUS).

Os procedimentos de Alta Complexidade são especificados como um conjunto de procedimentos envolvendo alta tecnologia e alto custo, no sentido de propiciar à população acesso a serviços qualificados, integrando-os aos demais níveis de atenção à saúde: atenção básica e de média complexidade (Paim, 2009; Ministério da Saúde, 2009).

Ao tratar os procedimentos ambulatoriais de alta complexidade, isoladamente (excluindo os procedimentos de média complexidade e os medicamentos excepcionais), Solla e Chioro (2008) observaram que no Brasil, em 2005, 42\% desses procedimentos foram realizados por prestadores públicos e $58, \%$ o foram por privados (22\% privados lucrativos e $37 \%$ não lucrativos). Em 2010, o predomínio dessa oferta por unidades privadas ampliou-se para 78\% (36\% lucrativas e $42 \%$ não lucrativas). Cabe salientar que nesse ano os procedimentos mais realizados pelo setor privado foram: tomografia computadorizada $(51,7 \%)$, radiologia intervencionista $(65,4 \%)$, ressonância magnética $(73,4 \%)$, medicina nuclear in vivo $(76,2 \%)$, terapia renal substitutiva $(94,4 \%)$, sendo, portanto, a nefrologia o segmento de menor $(5,5 \%)$ oferta pública.

Até recentemente, o modelo de alocação de recursos financeiros na área da atenção especializada vinha sendo totalmente centralizado na lógica de pagamento por procedimento, uma herança da assistência médica previdenciária hegemônica no Brasil durante as décadas de 1970 e 1980 (Solla e Chioro, 2012). 
Tal modelo pode reforçar uma estratégia perversa de produção sem critérios, como a realização de procedimentos desnecessários onerando o sistema de saúde e, muitas vezes, incentivando a corrupção, como ocorreu com as chamadas Unidades de Serviço (US) no passado, quando a contratação de serviços privados de saúde era feita por meio de credenciamento e remunerada por US, forma de pagamento que Carlos Gentile de Mello denominava de "fator incontrolável de corrupção". Isso porque, como os preços tabelados em US eram baixos, os serviços prestadores inventavam pacientes ou ações que não haviam sido executadas, ou ainda selecionavam aquelas que geravam melhor remuneração, no sentido de transformar a prática médica em uma atividade lucrativa (Escorel, 2008).

Nesse sentido, Solla e Chioro (2012) e Mendes (2011) salientam que o sistema de pagamento, fortemente centrado no pagamento por procedimentos, sinaliza para os prestadores de serviços que ofertem mais serviços e serviços mais caros para maximizar seus ganhos, deixando assim de ofertar serviços pela lógica da necessidade. Ao estimular a oferta do "máximo de cuidado possível", em vez do cuidado necessário, esse sistema desestimula a oferta de intervenções voltadas para a promoção e a manutenção da saúde.

Os achados acerca da produção e dos gastos com a assistência de média e, principalmente, de alta complexidade, assim como a massiva participação do setor privado/filantrópico nessa provisão, reforçam a necessidade, já abordada, de um efetivo complexo regulador.

Desse ponto de vista, Oliveira e Elias (2012) afirmam que a regulação tem sido uma resposta potencial para o enfrentamento de questões de diferentes naturezas nos sistemas de saúde, tais como a produção privada, o financiamento e a oferta de serviços de saúde. Dessa forma, é necessária uma regulação adequada por parte do Estado para garantir que prevaleça o interesse público, independentemente da magnitude da participação privada no SUS.

Soma-se a isso o fato de que, no contexto da regionalização, a assistência de média e alta complexidade tem sido um dos grandes desafios à maioria dos gestores municipais. Nesse sentido, além da regulação, os instrumentos de gestão são imprescindíveis, ressaltando-se o papel da Programação Pactuada e Integrada (PPI). A PPI é o instrumento de pactuação entre gestores municipais para o uso racional dos recursos assistenciais. Este processo deve se dar sob a coordenação da secretaria estadual de saúde e com apreciação pelas comissões intergestores regionais. Os gestores de municípios mais carentes devem negociar com os demais a utilização de serviços de saúde de que não dispõem, objetivando garantir a integralidade da atenção à saúde da população de sua região (Paim, 2009).

No que tange à assistência de média e alta complexidade, a questão do financiamento é outro ponto de estrangulamento. Para Mendes (2011), sem um alinhamento dos incentivos financeiros, por meio da superação do sistema de pagamento por procedimentos, e sem a alteração dos modelos de atenção à saúde praticados no SUS, quaisquer recursos adicionais podem ser rapidamente apropriados por prestadores de serviços mais bem posicionados na arena política da saúde, sem que gerem, necessariamente, benefícios para os cidadãos.

Os investimentos empreendidos na área de atenção especializada, principalmente com recursos provenientes do orçamento federal e do programa de investimentos do SUS (Refor- 
sus), ampliaram a rede de serviços especializados sem planejamento, de maneira que uma enorme quantidade de serviços de atenção especializada públicos e filantrópicos foi entrando em funcionamento sem a devida cobertura de recursos de custeio para acolher o impacto dessa ampliação no teto financeiro de estados e municípios (Solla e Chioro, 2012).

No período de 1994 a 2002 houve um grave "desfinanciamento" do sistema. Não houve correção sistemática dos tetos financeiros de estados e municípios. Os recursos foram sendo direcionados para ações de alta complexidade, ficando os procedimentos de média complexidade sem a devida atualização. Nesse período, os procedimentos de média complexidade acumularam um déficit de $110 \%$, ao mesmo tempo que houve elevação dos custos com medicamentos e insumos da ordem de 129\%, medidos pela inflação Fipe-saúde. Portanto, a tabela de procedimentos do SUS foi sofrendo diversas distorções. A pressão pela incorporação de novas tecnologias, medicamentos de alto custo (que se transformaram em "procedimentos" da tabela), órteses e próteses dominou a atenção dos gestores sem haver estudos prévios de avaliação tecnológica que subsidiasse a incorporação de novos procedimentos/tecnologias, estudos de custo/efetividade; custo/benefício e impacto financeiro (Solla e Chioro, 2012).

Os mesmos autores referem ainda que o Ministério da Saúde enfrentou tal situação, entre os anos de 2003 e 2005, tomando diversas medidas, como os reajustes anuais das tabelas, principalmente nos procedimentos com valores mais defasados como os da média complexidade; ampliação de tetos privilegiando estados com renda per capita mais baixa; financiamento de novas políticas de repasse globais; e implantação progressiva de contratos de metas com pagamentos globais (ou mistos), retirando um segmento importante para os hospitais, que é o pagamento por procedimento.

O que é referido por Solla e Chioro (2012), acerca da menor valorização dos procedimentos de média complexidade em relação aos de alta complexidade, materializa-se na região da Baixada Cuiabana. Neste território, os quase 7 milhões de procedimentos de média complexidade redundaram em um gasto aproximado de 53 milhões de reais, enquanto 300 mil procedimentos de alta complexidade geraram um gasto de 41 milhões de reais.

Machado (2002) também corrobora o exposto por Solla e Chioro (2012), quando salienta que desde a NOB-SUS 01/93 vem ocorrendo uma progressiva substituição do pagamento dos prestadores por produção diretamente pelo administrador federal, pelas transferências automáticas e em bloco de recursos federais aos gestores estaduais e municipais para que assumam as funções de gerência do sistema. Tal mudança poderia levar a uma redução da importância das tabelas nacionais de remuneração dos serviços ambulatoriais e hospitalares, caso esses gestores pudessem utilizar livremente os recursos transferidos. Para essa autora, até o início dos anos 2000 essas tabelas representavam instrumentos importantes de orientação da remuneração dos serviços pelos seguintes motivos: mais de 30\% dos recursos federais ainda são pagos diretamente pelo gestor federal aos prestadores, segundo produção aprovada pelas esferas estaduais; mesmo os administradores municipais e os estaduais utilizam a tabela nacional como referência para a remuneração dos prestadores, ainda que a prática de complemento da tabela seja comum em algumas regiões do país; esses administradores têm a obrigação de intensificar os sistemas de informações nacionais de produção, o SIA-SUS e o 
SIH-SUS, que são organizados com base no conteúdo dessas tabelas. Pelo menos os dois últimos motivos continuam válidos atualmente.

Tais aspectos sinalizam ainda a existência da vinculação entre produção e remuneração no SUS, apesar das alterações da política federal de repasse financeiro. Entende-se que tais mudanças são lentas e necessitam de esforços coletivos envolvendo pactuação (negociação) entre gestores, uma efetiva regulação e controle social do sistema para sua superação.

\section{Considerações finais}

A regulamentação do SUS explicita que o setor privado/filantrópico deve complementar os serviços de saúde quando houver impossibilidade do setor público em oferecê-los. A situação na região de saúde da Baixada Cuiabana aponta o oposto: o setor privado/filantrópico que deveria ser complementar constitui-se o principal provedor de serviços de assistência ambulatorial de alta e média complexidade do SUS.

Cuiabá detém o maior número de estabelecimentos ambulatoriais de média e alta complexidade na região de saúde da Baixada Cuiabana, sendo referência regional e estadual em saúde, o que destaca sua importância na oferta de serviços de saúde à população. Porém, observa-se uma forte relação entre o público e o privado dentro do SUS nesse município, sendo o setor privado/filantrópico majoritário na oferta e principalmente na produção de serviços dessa natureza, bem como nos pagamentos relacionados a essa produção. Tal fato sugere a possibilidade de que o setor privado/filantrópico detenha expressivo poder político e poder de pressão, quando da negociação de seus contratos e da remuneração por seus serviços, no âmbito do SUS.

As evidências apresentadas sinalizam para a necessidade de uma maior articulação entre gestores municipais da região da Baixada Cuiabana e o gestor estadual com vistas: à regulação (inclusive a contratualização) da participação privada/filantrópica nos serviços de saúde do SUS; ao fortalecimento e à qualificação do processo de programação pactuada e integrada; à maior abertura à participação social neste sistema. Essas seriam algumas iniciativas com potencial para avançar em direção a um modelo de atenção focado no perfil epidemiológico e nas necessidades da população e não na oferta de serviços, assegurando o acesso dos usuários de forma equitativa e de qualidade a esses serviços.

\section{Referências}

BAHIA, Ligia. A dérmache do privado e público no sistema de atenção à saúde no Brasil em tempos de democracia e ajuste fiscal, 1988-2008. In: MATTA, Gustavo C.; LIMA, Júlio C. F. (Org.). Estado, sociedade e formação profissional em saúde: contradições e desafios em 20 anos de SUS. Rio de Janeiro: Fiocruz; ESPJV, 2008. cap. 4, p. 123-178. 
BAHIA, Ligia. Mudanças e padrões das relações público-privado: seguros e planos de saúde no Brasil. Tese (doutorado) — Escola Nacional de Saúde Pública, Fundação Oswaldo Cruz, Rio de Janeiro, 1999.

BRASIL. Constituição da República Federativa do Brasil, 1988. Disponível em: <www.planalto.gov. br/ccivil_03/constituicao/constituicao.htm\#adct>. Acesso em: 23 jan. 2013.

CAMPOS, Gastão W. S. Políticas sociais: o público e o privado na saúde brasileira. Le Monde Diplomatique, v. 1, n. 8, p. 28-29, mar. 2008. Disponível em: <www.diplomatique.uol.com.br/artigo>. Acesso em: 19 abr. 2011.

CAVALCANTI, Alexandre G. A regulação assistencial no Sistema Único de Saúde: o caso da Central de Regulação de Cuiabá/MT. Dissertação (mestrado) - Universidade Federal de Mato Grosso, Instituto de Saúde Coletiva, Cuiabá, 2011.

CNES. Cadastro nacional de estabelecimentos de saúde. Disponível em: <www.datasus. gov.br>. Acesso em: 22 ago. 2012.

CONASS. Conselho Nacional de Secretários de Saúde. Assistência de média e alta complexidade no SUS. Brasília, 2007.

DAVANZO, Aurea M. Q.; FONSECA, Rinaldo B. Estudo para o regionalismo do SUS. In: NEGRI, Barjas; VIANA, Ana Luíza d’Á. (Org.). O Sistema Único de Saúde em dez anos de desafio. São Paulo: Sobravime; Cealag, 2002.

ESCOREL, Sarah. História das políticas de saúde no Brasil de 1964 a 1990: do golpe militar à reforma sanitária. In: GIOVANELLA, Lígia et al. (Org.). Políticas e sistema de saúde no Brasil. Rio de Janeiro: Ed: Fiocruz, 2008. p. 385-434.

FARIAS, Sidney F. et al. A regulação no setor público de saúde no Brasil: os (des) caminhos da assistência médico-hospitalar. Revista Ciência \& Saúde Coletiva, Rio de Janeiro, v. 16, n. supl. 1, p. 1043-1053, 2011. Disponível em: <www.scielo.br/scielo.php?pid=S1413-81232011000700037\&script=sci_arttext $>$ Acesso em: 9 nov. 2012.

FAVERET FILHO, Paulo; OLIVEIRA, Pedro J. de. A universalização excludente: reflexões sobre as tendências do sistema de saúde. Planejamento e Políticas Públicas, Brasília, n. 3, p. 139-162, jun. 1990.

FLEURY, Sonia. El desafio da gestión de las rede de políticas. Revista Instituições y Desarrollo, n. 12-13, p. 221-247, dez. 2002.

GLOBEKNER, Osmir A. A saúde entre o público e o privado: o desafio da alocação social dos recursos sanitários escassos. Curitiba: Juruá, 2011.

GOTTEMS, Leila B. D. et al. Análise da relação dos serviços de média complexidade com a rede básica do DF e entorno: buscando prioridades para ampliar acessos e resolubilidades. Relatório final da pesquisa. Brasília-DF: Universidade de Brasília; Universidade Católica de Brasília; Universidade Federal de Minas Gerais, 2008. Disponível em: <www.nesprom.universidadevirtual.br>. Acesso em: 8 abr. 2013. 
IBGE. Instituto Brasileiro de Geografia e Estatística. Censo demográfico de 2010 no estado de Mato Grosso. Disponível em: <www.ibge.gov.br/estadosat/perfil.php?sigla=mt\#>. Acesso em: 20 mar. 2013.

LEITE, Jacqueline D. B. L.; SOUZA, Hellen F.; NASCIMENTO, Eliana, C. V. Contratualização na saúde: proposta de um Contrato Único para os programas no estado de Minas Gerais. Revista de Administração de Saúde, v. 12, n. 46, p. 15-22, 2010.

MACHADO, Cristiani V. Regulação da Política Nacional de Saúde: estratégias e tendências. In: NEGRI, Barjas; VIANA, Ana Luiza d'Á. O Sistema Único de Saúde em dez anos de desafios. São Paulo: Sobravime; Cealag, 2002. p. 505-526.

MATOS, Carlos Alberto de; POMPEU, João Cláudio. Onde estão os contratos? Análise da relação entre os prestadores privados de serviços de saúde e o SUS. Revista Ciência \& Saúde Coletiva, v. 8, n. 2, p. 629-643, 2003. Disponível em: <www.scielo.br/pdf/csc/v8n2/a23v08n2.pdf>. Acesso em: 6 set. 2012.

MENDES, Eugênio V. As redes de atenção à saúde. Brasília, DF: Organização Pan-Americana da Saúde; Organização Mundial da Saúde; Conselho Nacional de Secretários de Saúde, 2011.

MENDES, Eugênio V. O SUS que temos e o SUS que queremos: uma agenda. Revista Mineira de Saúde Pública, v. 3, n. 4, p. 4-26, jan./jun. 2004. Disponível em: <www.esp.mg.gov.br/wp-content/ uploads/2009/07/revista04.pdf>. Acesso em: 4 set. 2012.

MENDES, Eugênio V. Os grandes dilemas do SUS. Salvador: Casa da Qualidade, 2001.

MENICUCCI, Telma M. G. A reforma sanitária brasileira e as relações entre o público e o privado. In: SANTOS, Nelson R. dos; AMARANTE, Paulo D. C. (Org.). Gestão pública e relação público privado na saúde. Rio de Janeiro: Cebes, 2010. cap. 10, p. 180-197.

MINISTÉRIO DA SAUUDE. O SUS de A a Z: garantindo saúde nos municípios. Brasília, 2009.

NORONHA, José C.; SANTOS, Isabela S.; PEREIRA,Telma R. Relações entre o SUS e a saúde suplementar: problemas e alternativas para o futuro do sistema universal. In: SANTOS, Nelson R.; AMARANTE, Paulo D. C. (Org.). Gestão pública e relação público privado na saúde. Rio de Janeiro: Cebes, 2010. cap. 9, p. 152-179.

OLIVEIRA, Robson R. de; ELIAS, Paulo E. M. Conceitos de regulação em saúde no Brasil. Revista de Saúde Pública, v. 46, n. 3, p. 571-576, 2012.

PAIM, Jairnilson S. O que é o SUS. Rio de Janeiro: Fiocruz, 2009.

PUSTAI, Odaci J. O sistema de saúde no Brasil. In: DUNCAN, Bruce B.; SCHIMIDT, Maria Ines; GIUGLIANI, Elsa R. J. (Org.). Medicina ambulatorial: condutas de atenção primária baseadas em evidências. Porto Alegre: Artmed, 2004. p. 69-75.

SANTOS, Fausto P. dos; MERHY, Emerson E. A regulação pública da saúde no Estado brasileiro - uma revisão. Revista Interface - Comunicação, Saúde, Educação, v. 10, n. 19, p. 25-41, jan./ jun. 2006. 
SANTOS, Lenir. Administração pública e a gestão da saúde. In: SANTOS, Nelson R. dos; AMARANTE, Paulo D. C. (Org.). Gestão pública e relação público privado na saúde. Rio de Janeiro: Cebes, 2010. cap. 4, p. 68-86.

SANTOS, Lenir. Sistema Único de Saúde: os desafios da gestão interfederativa. Campinas, SP: Saberes, 2013.

SIA-SUS. Sistema de Informações Ambulatoriais do SUS. Disponível em: <www.datasus. gov.br> Acesso em: 23 ago. 2012.

SILVA, Silvio F. da. A construção do SUS a partir do município. São Paulo: Hucitec, 1996.

SOLLA, Jorge J. S. P. Avanços e limites da descentralização no SUS e o "Pacto de Gestão". Revista Baiana de Saúde Pública, v. 30, n. 2, p. 332-348, jul./dez. 2006.

SOLLA, Jorge; CHIORO, Arthur. Atenção ambulatorial especializada. In: GIOVANELLA, Lígia et al. (Org.). Políticas e sistemas de saúde no Brasil. Rio de Janeiro: Fiocruz, 2008. cap. 17, p. 627-663.

SOLLA, Jorge; CHIORO, Arthur. Atenção ambulatorial especializada. In: GIOVANELLA, Lígia et al. (Org.). Políticas e sistemas de saúde no Brasil. 2. ed. Rio de Janeiro: Fiocruz, 2012. cap. 17, p. 547-576.

SPEDO, Sandra M. Desafios para implementar a integralidade da assistência à saúde no SUS: estudo de caso no município de São Paulo. Tese (doutorado) — Faculdade de Saúde Pública, Universidade de São Paulo, 2009.

VIANA, Ana Luiza d'Á. et al. Pesquisa Nacional de Avaliação das CIB. Brasília: Ministério da Saúde, 2010. Disponível em: <http://bvsms.saude.gov.br/bvs/descentralizacao/index.php>. Acesso em: 15 ago. 2012.

Cátia Maria C. Romano é doutoranda do Programa de Pós-Graduação em Ciências da Saúde da Universidade Federal de Mato Grosso e professora adjunto IV da Universidade Federal da Bahia. E-mails: catia. romano4@gmail.com; romano@ufba.br.

João Henrique G. Scatena é doutor em saúde pública pela Universidade de São Paulo, professor associado do Instituto de Saúde Coletiva da Universidade Federal de Mato Grosso. E-mail: jscatena@ufmt.br. 